\title{
Microscopic Analysis of Heterogeneous Nucleation of
} Nanoparticle Superstructures

Ann-Kathrin Göppert, Guillermo González-Rubio, Helmut Cölfen*

Physical Chemistry, University of Konstanz, Universitätsstraße 19, 78457 Konstanz, Germany

*E-Mail: helmut.coelfen@uni-konstanz.de 


\section{Table of Contents}

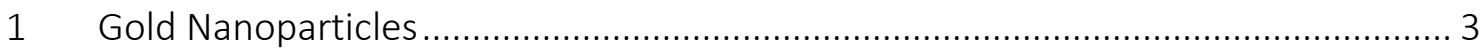

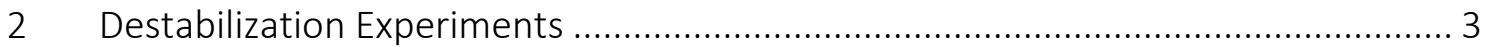

3 Preparation of Gold Nanoparticle Solutions ............................................................ 4

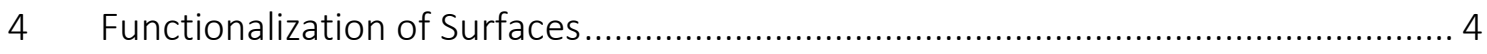

$5 \quad$ Heterogeneous Nucleation of Gold Nanocube Superstructures .......................... 6

6 Destabilization inside a Functionalized Cuvette ................................................... 7

$7 \quad$ Development of the Experimental Setup .......................................................... 9

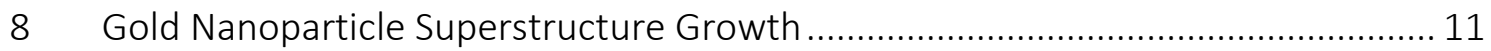

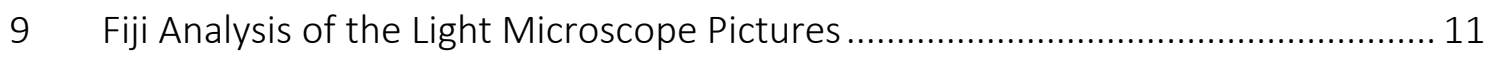

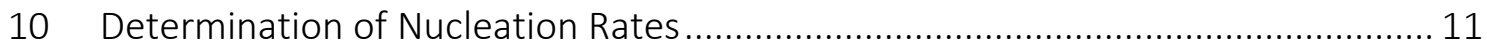

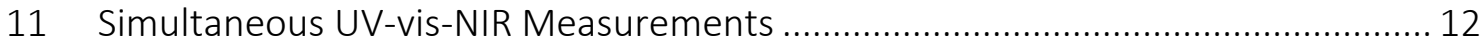

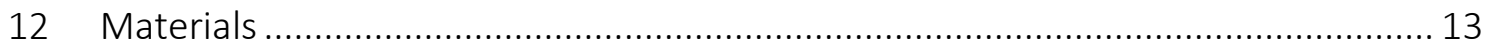

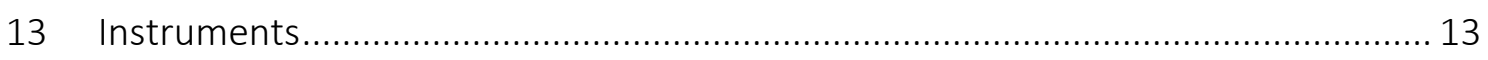

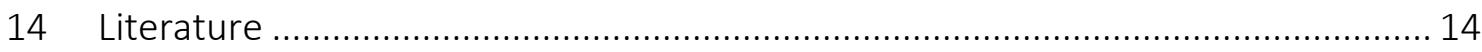




\section{Gold Nanoparticles}

A)

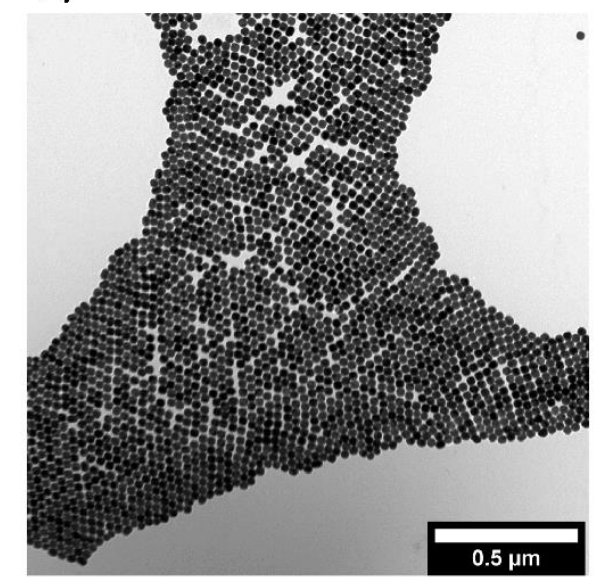

B)

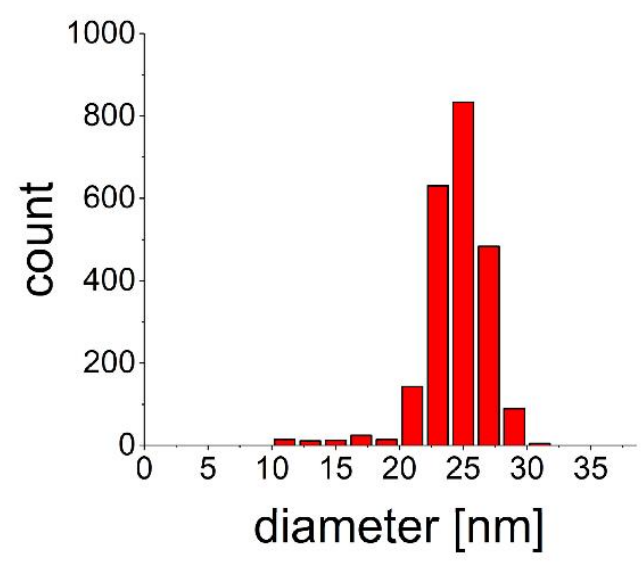

Figure S 1: A) TEM picture of the used gold nanoparticles. B) Size distribution of the gold nanoparticles.

\section{Destabilization Experiments}

\subsection{Destabilization of Nanoparticle Solutions}

To destabilize the stock solution of gold nanoparticles ethanol was added to an amount of 12 to 38 vol.-\%.

Table S1. Summary of the nucleation experiments with gold nanoparticles with the experimental conditions.

\begin{tabular}{|c|c|c|c|c|c|}
\hline experiment & $\begin{array}{l}\text { reaction } \\
\text { vessel }\end{array}$ & surface & $\begin{array}{c}\text { surface } \\
\text { function- } \\
\text { nalization }\end{array}$ & $\begin{array}{l}\text { EtOH content } \\
\text { [Vol.-\%] }\end{array}$ & $\begin{array}{c}\text { surface/ } \\
\text { volume ratio } \\
{[1 / \mathrm{cm}]}\end{array}$ \\
\hline $\begin{array}{l}\text { homogeneous } \\
\text { nucleation }\end{array}$ & cuvette & - & - & 38 & - \\
\hline \multirow{3}{*}{$\begin{array}{l}\text { introducing } \\
\text { mica surface }\end{array}$} & \multirow{3}{*}{$\begin{array}{l}\text { micro test } \\
\text { tube }\end{array}$} & \multirow[t]{2}{*}{ mica } & PSS & 31 & 0,39 \\
\hline & & & fluorine & 31 & 0,39 \\
\hline & & - & - & 31 & - \\
\hline \multirow[t]{2}{*}{ kinetics } & cuvette & cuvette & PSS & 15 & 2,45 \\
\hline & cuvette & cuvette & fluorine & 15 & 2,45 \\
\hline \multirow{2}{*}{$\begin{array}{l}\text { light } \\
\text { microscopy }\end{array}$} & \multirow{2}{*}{$\begin{array}{l}\text { Hydropho- } \\
\text { bized cuvette }\end{array}$} & mica & PSS & 12 & 0,09 \\
\hline & & mica & fluorine & 12 & 0,09 \\
\hline
\end{tabular}

\subsection{Quenching the Destabilized Nanoparticle Solutions}

During or after the destabilization experiments the formed aggregates and remained single particles were stabilized again. Therefore, a CTAB solution was added to adjust the amount of CTAB to $2 \mathrm{mM}$. To analyze formed structures on the mica surfaces, these were washed thoroughly with water and dried with a nitrogen stream at RT. 


\section{Preparation of Gold Nanoparticle Solutions}

For the destabilization experiments a stock solution of the gold nanoparticles from chapter $\mathbf{1}$ gold nanoparticles was prepared. One batch of $50 \mathrm{ml}$ aqueous nanoparticle solution, with a concentration of $0,17 \mathrm{mM}$ gold nanoparticles, was centrifuged at 6000 RPM for 1 hour. The supernatant was separated and the concentrated nanoparticles in the sediment were transferred to a micro test tube. The concentrated nanoparticles were washed with a $2 \mathrm{mM} \mathrm{CTAB}$ aqueous solution and centrifuged at 6000 RPM for 20 minutes. The washing procedure was repeated for two times. The volume of the concentrated nanoparticle sediment was determined to know the current concentration of the gold nanoparticles. A solution of $0,02 \mathrm{mM} \mathrm{CTAB}$ in water was prepared. The concentrated nanoparticles were added to the CTAB solution to reach a concentration of $0,5 \mathrm{mM}$ of gold nanoparticles. Before using the stock solution of gold nanoparticles in experiments, the stability of the nanoparticles was checked in the UV-vis-NIR.

\section{Functionalization of Surfaces}

Before functionalization, mica surfaces were freshly cleaved using a cello tape. The Si wafers were cleaned in an ultrasonic bath for 10 minutes each in propan-2-ol, ethanol and acetone. The quartz cuvettes were cleaned in aqua regia for 1 hour and afterward cleaned thoroughly with water.

The different surfaces were plasma treated to generate free hydroxyl groups.

The surface functionalization was carried out using a vapor phase diffusion under a protective gas atmosphere. ${ }^{1} \mathrm{~A} 4 \mathrm{~L}$ desiccator was evacuated and flooded with nitrogen for two times. The surfaces were placed in the desiccator and it was evacuated and flooded with nitrogen for a third time to reach a humidity under $25 \% .60 \mu \mathrm{L}$ of the corresponding liquid silane (amine or fluor) and $20 \mu \mathrm{L}$ of 1,1Diisopropylethylamine were added to the desiccator in separate beakers. The desiccator was evacuated for 30 seconds, sealed afterward and left for 2 hours (amine functionalization) or overnight (fluorine functionalization) at RT. Afterwards the functionalized surfaces were baked for 2 hours at 150 ${ }^{\circ} \mathrm{C}$ under ambient conditions.

For a functionalization with PSS the amine-functionalized surfaces were given into a solution of $1 \%$ PSS in water for one hour. Afterward the surfaces were cleaned thoroughly with water and dried at $50{ }^{\circ} \mathrm{C}$.

All functionalized surfaces were used in a period of one to two days after the functionalization.

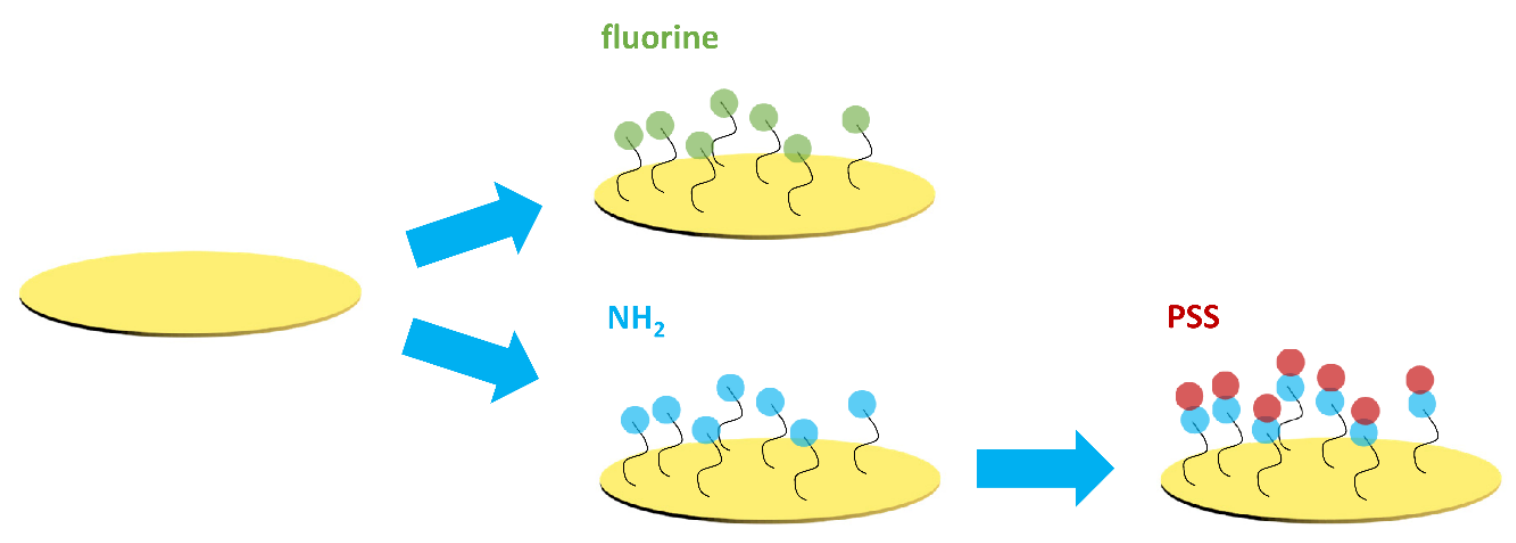

Figure S 2. Scheme for the functionalization of the surfaces. In the first step either the fluorine or amine are coupled with a vapour phase diffusion. In a second step the PSS can be coupled to the amine functionalization. 
Table S2. Characterization of the functionalized mica surfaces.

\begin{tabular}{|l|l|l|l|l|}
\hline & Mica & Mica-amine & Mica-PSS & Mica-fluorine \\
\hline contact angle water $\left[^{\circ}\right]$ & $13,26 \pm 0,25$ & $47,29 \pm 0,13$ & $45,64 \pm 0,98$ & $73,71 \pm 1,40$ \\
\hline $\begin{array}{l}\text { contact angle } \\
\text { diiodomethane }\left[^{\circ}\right]\end{array}$ & $52,88 \pm 0,11$ & $39,42 \pm 0,10$ & $50,67 \pm 0,37$ & $74,49 \pm 2,87$ \\
\hline $\begin{array}{l}\text { free surface energy } \\
{[\mathrm{mN} / \mathrm{m}]}\end{array}$ & $72,62 \pm 0,14$ & $59,48 \pm 0,13$ & $57,46 \pm 0,83$ & $33,17 \pm 2,73$ \\
\hline dispersive part [mN/m] & $32,65 \pm 0,06$ & $39,90 \pm 0,05$ & $33,90 \pm 0,21$ & $20,40 \pm 1,56$ \\
\hline polar part [mN/m] & $39,97 \pm 0,08$ & $19,58 \pm 0,08$ & $23,56 \pm 0,62$ & $12,77 \pm 1,17$ \\
\hline roughness $\mathrm{R}_{\mathrm{ms}}[\mathrm{nm}]^{(1)}$ & 0,371 & 0,440 & 0,211 & 0,146 \\
\hline roughness $\mathrm{R}_{\mathrm{a}}[\mathrm{nm}]^{(1)}$ & 0,288 & 0,345 & 0,166 & 0,117 \\
\hline${\text { thickness }[\mathrm{nm}]^{(2)}}^{-2}$ & 0,96 & 1,94 & 0,12 \\
\hline
\end{tabular}

(1) $R_{m s}=$ root mean square of the roughness height and $R_{a}=$ surface roughness value $R_{\text {max }} / 4$ were determined with $A F M$ over a surface of $1 \times 1 \mu \mathrm{m}$.

(2) The layer thickness was determined using ellipsometry. A polished Si wafer was used as surface since mica cannot be used in this method because of its birefringence. 


\section{Heterogeneous Nucleation of Gold Nanocube Superstructures}

A)
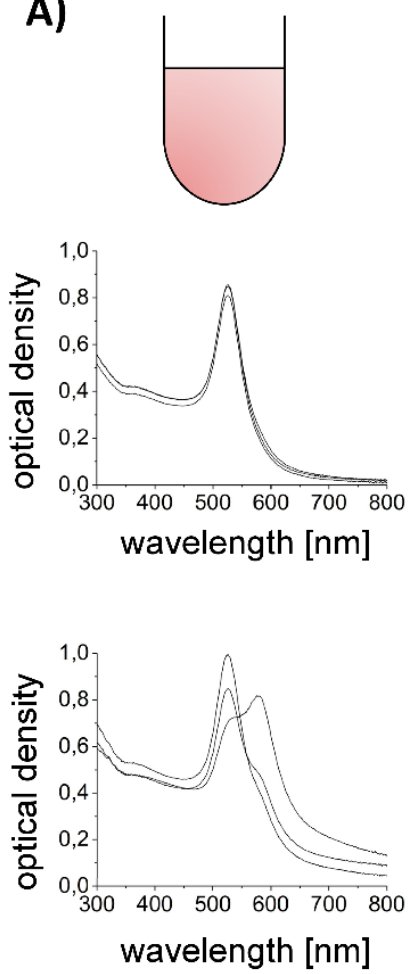

B)
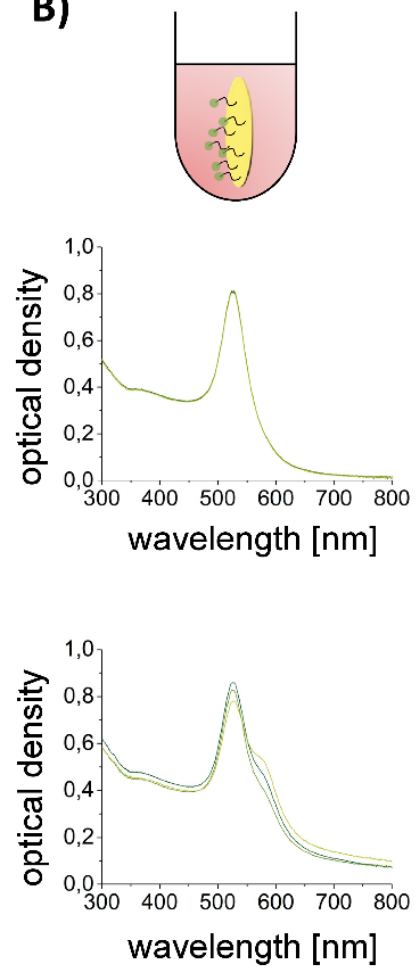

C)
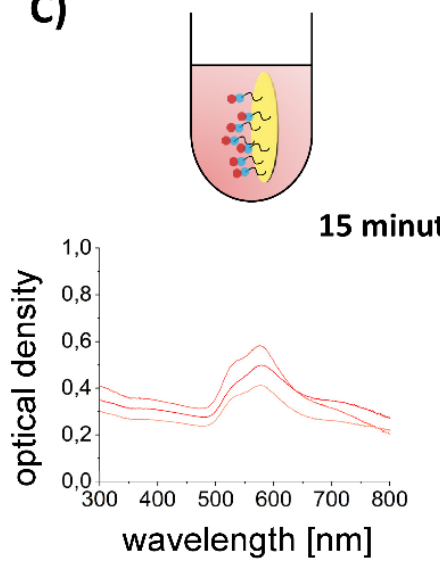

2 hours

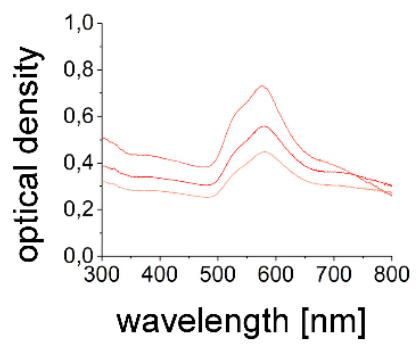

Figure S 3. A gold nanoparticle solution was destabilized with EtOH in a micro test tube without and with the presence of different functionalized mica surfaces. Aliquots at different time points were analyzed with UV-vis-NIR spectroscopy to compare the destabilization of the nanoparticle solution. A) shows the destabilization of the gold nanoparticle solution without the presence of an additional surface after 15 minutes (upper row) and after 2 hours (bottom row). The gold nanoparticles start to aggregate slowly, which is indicated by the arising peaks at higher wavelengths. B) shows the destabilization with the presence of a fluorine functionalized surface after 15 minutes (upper row) and after 2 hours (bottom row). The solution behaves comparable to the solution without an additional surface. This indicates that the gold nanoparticles do not interact with the fluorine surface. $\mathrm{C}$ ) shows the destabilization with the presence of a PSS functionalized surface after 15 minutes (upper row) and after 2 hours (lower row). The gold nanoparticles destabilize much faster in the presence of the PSS functionalized surface. This indicates an interaction of the gold nanoparticles with the PSS functionalized surface. 


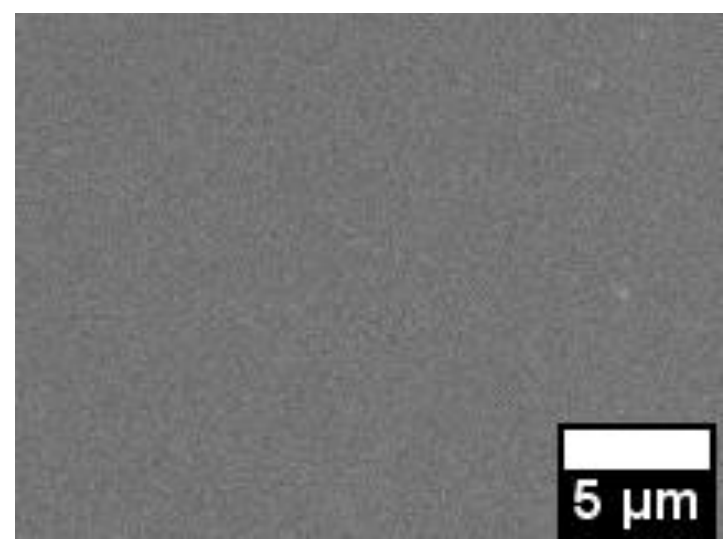

Figure S 4. SEM image of a hydrophobized mica surface after 24 hours of heterogeneous nucleation of gold nanocube superstructures. The surface is empty and no gold nanocube superstructures formed on the surface.

\section{Destabilization inside a Functionalized Cuvette}

A)

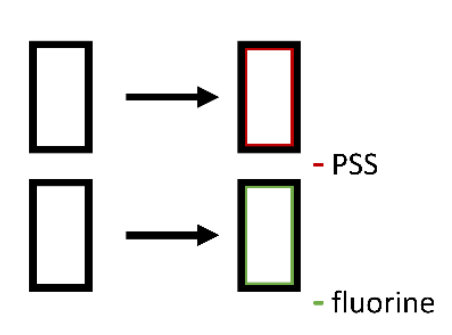

B)

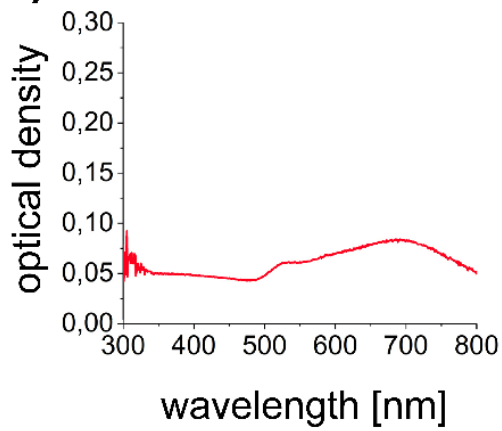

C)

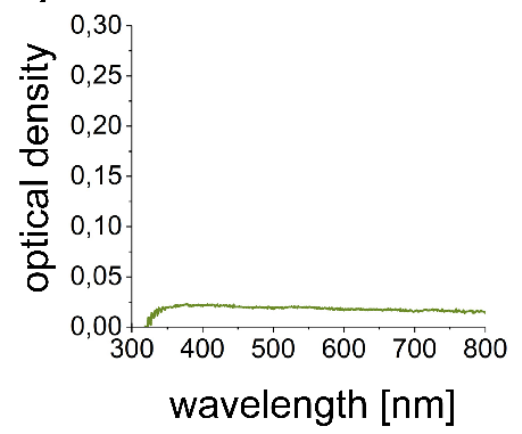

Figure S 5. Characterization of heterogeneous nucleation of gold nanocube superstructures with different functionalized quartz cuvettes. A) Schematic representation of the functionalization of quartz cuvettes used to investigate the heterogeneous nucleation of nanocube superstructures in solution via UV-vis-NIR spectroscopy. B) The UV-vis-NIR spectra of the gold nanoparticle superstructures formed on the PSS functionalized surface. C) The UV-vis-NIR spectra for the hydrophobized surface, where no gold nanoparticle superstructures formed. 


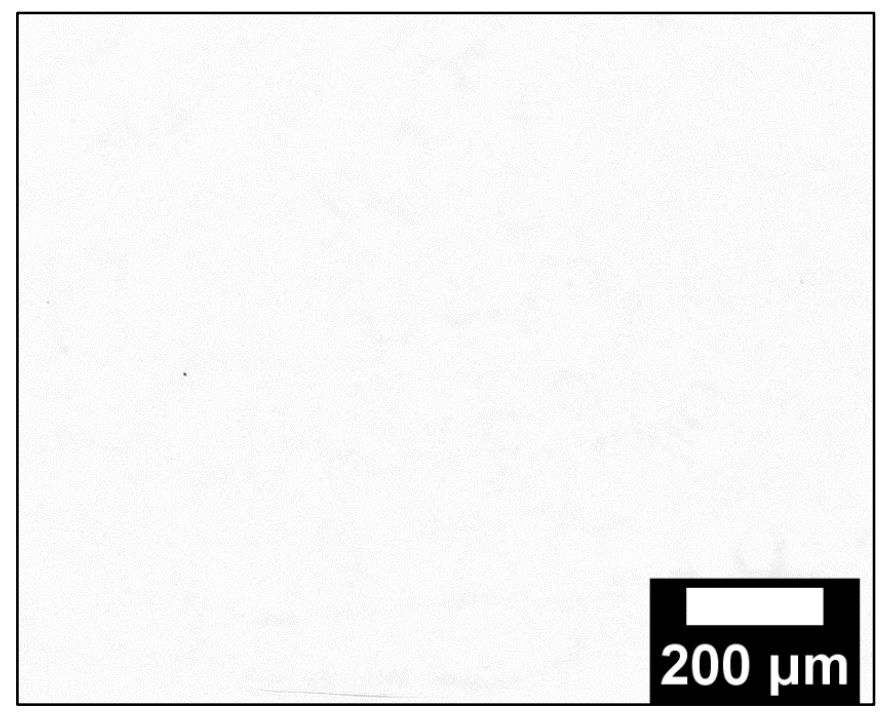

Figure S 6. Light microscopy image of the hydrophobized cuvette surface after the nucleation process of gold nanoparticle superstructures. 


\section{Development of the Experimental Setup}

We aimed to destabilize the gold nanoparticles in a controlled way, obtain heterogeneous nucleation of nanoparticle superstructures on the substrates but prevent nucleation in solution or at other phase boundaries.

To realize this experiment we tried different triggers for the nanoparticle destabilization and experiment set-ups. At first, the surface for heterogeneous nucleation has to be on top of the set-up to distinguish between heterogeneously formed structures on the surface and homogeneously formed structures that sediment down (Figure S8 $\mathbf{A}$ and $\mathbf{B}$ ). The first trigger we used to destabilize the nanoparticle solution was an increase in the concentration via drying. Therefore, we constructed a climate chamber, which allows controlling the temperature and humidity in experiments under the light microscope (Figure S7 A). With this chamber it is possible to evaporate a drop of an aqueous nanoparticle solution under low humidity. With the evaporation of the water, the concentration of the nanoparticles is increased and the nucleation is triggered. The evaporation can be slowed down after a while by raising the humidity so that the volume stays almost constant (Figure S 7B). Some problems arise with a drying drop, as it offers ill-defined conditions. First the coffee-ring effect leads to a diffusion of the nanoparticles resulting in nucleation at the solvent air interface. ${ }^{2-4}$ Thus, the particle concentration varies inside the solution and the nucleation doesn't take place at the desired mica surface inside the volume (Figure S8 $\mathbf{C}$ ). Furthermore, an evaporating drop leads to temperature differences inside the solution. Hence, it was not possible to achieve individual nucleation of the nanoparticles on the substrates.
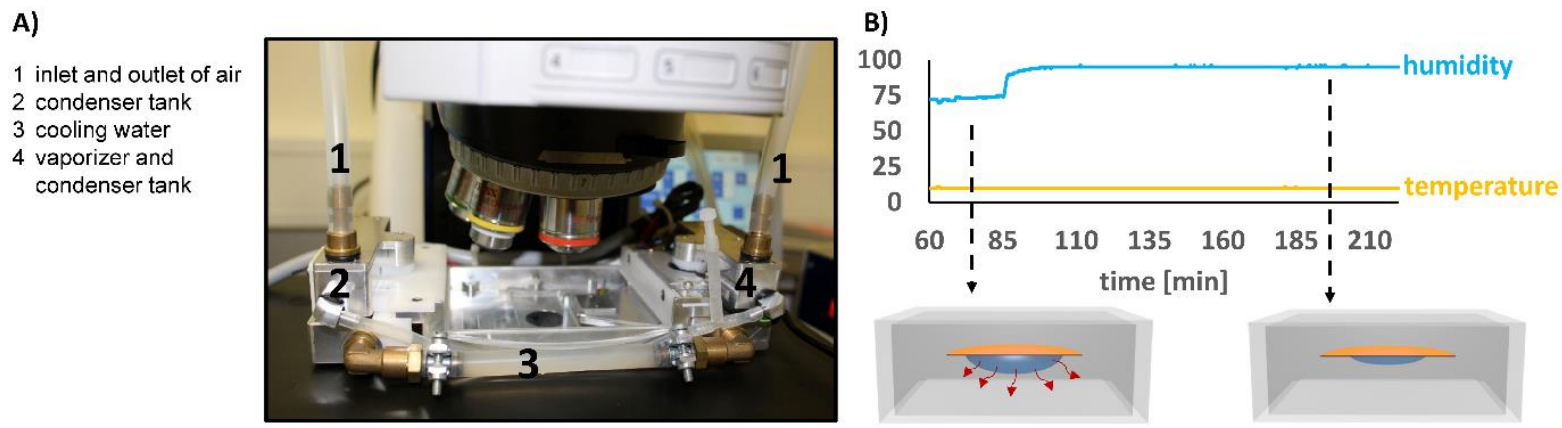

Figure S 7. A) Climate chamber which allows adjusting the temperature and humidity during experiments under the light microscope. B) With the climate chamber an experiment is possible where controlled evaporation of a drop under low humidity takes place. With increasing the humidity the evaporation is slowed down.

The second trigger we planned to use for nanoparticle destabilization was the change of the temperature. Unfortunately, we were not able to destabilize our nanoparticle systems with this method either. This approach would most likely work with temperature switching ligands, but the complex ligands would not be comparable with our desired variation in the surface chemistry of the nanoparticles ${ }^{5}$. Another possible trigger like gas diffusion, which is used to synthesise mesocrystals, is also not compatible with our system, since the nanoparticles are destabilized at the arising solventsolvent interface and not at the surface of our substrates. ${ }^{6}$

Finally, we used a closed setup (Figure S8 D) with mica on top to observe solely heterogeneous nucleation at the surface. The mica is glued on the glass with a hydrophobic seal spacer and the glass walls are functionalized with the hydrophobic (Heptadecafluoro-1,1,2,2-tetrahydrodecyl)triethoxysilane to prevent interactions between the nanoparticles and the walls. 
A)

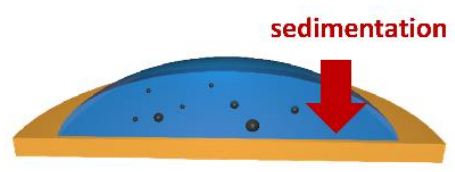

B)

heterogeneous

homogeneous
C)

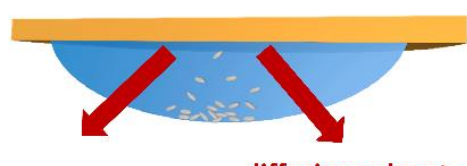

diffusion solventair interface

D) hydrophobic seal spacer

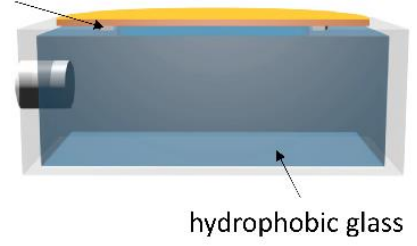

Figure S 8. A) An experiment carried out as a lying drop results in the sedimentation of homogeneously nucleated particles. A differentiation between heterogeneous or homogeneous nucleation is not possible. B) In an experiment carried out as a hanging drop homogeneously nucleated aggregates sediment down and a differentiation is possible. C) In an experiment with a drying drop, the particles diffuse with the solvent to the solvent-air interface where a maximum in the particle concentration leads to nucleation. D) The actually used system with the mica surface on top to observe only heterogeneous nucleation. The system is sealed without a solvent-air interface and the other surfaces are covered with a hydrophobic fluorine layer to prevent interaction with the particles. 


\section{Gold Nanoparticle Superstructure Growth}

See supporting video 1.
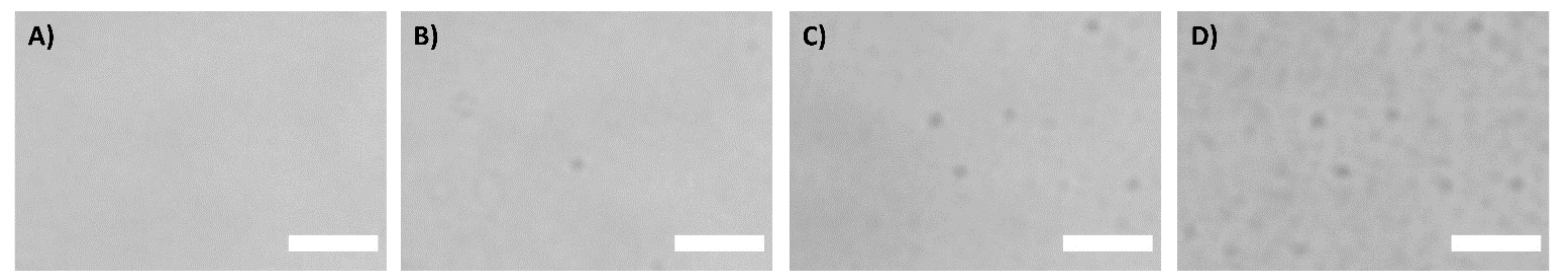

Figure S 9. Gold nanoparticle superstructure growth. Original light microscope images of growing gold nanocube mesocrystal nuclei on a PSS functionalized mica surface after A) 0, B) 11, C) 18 and D) 30 minutes. The scale bar is $10 \mu \mathrm{m}$.

\section{Fiji Analysis of the Light Microscope Pictures}

During the experiments light microscope pictures were taken every 60 respectively 30 seconds and processed with Fiji. After separating the timespan with nuclei growth a trainable weka segmentation was applied, differentiating between nuclei on the surface, background and moving aggregates in solution. The classifier results were given out as probabilities and the canal for the nuclei was used to count their number. Therefore, a threshold (MaxEntropy) was applied and the number of nuclei was counted by using the analyze particles function (size $=10-$ infinity pixel).

\section{Determination of Nucleation Rates}

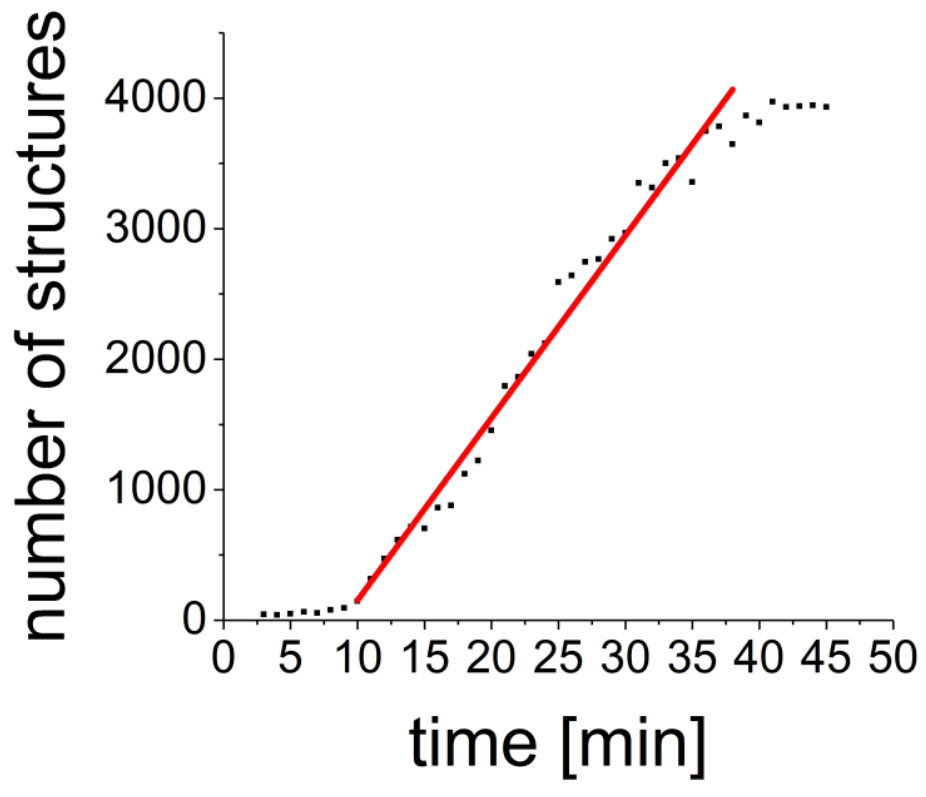

Figure S 10. Plot of the counted structures obtained from the Fiji analysis against the experiment time. We were able to detect the first nuclei growth after 10 minutes. The slope of the graph corresponds to the nucleation rate. After around $\mathbf{4 0}$ minutes no more nuclei appear. In the further analysis, only the growth periods in the plots were used.

To determine the nucleation rates, we plotted the counted number of structures against the time (Figure S10). Here the nucleation rate is the slope of the graph during the growth period. We calculated the slope by putting a linear fit through the data points. The adjusted R-square value of Figure $\mathbf{S 1 0}$ is 0,97902 . 


\section{Simultaneous UV-vis-NIR Measurements}

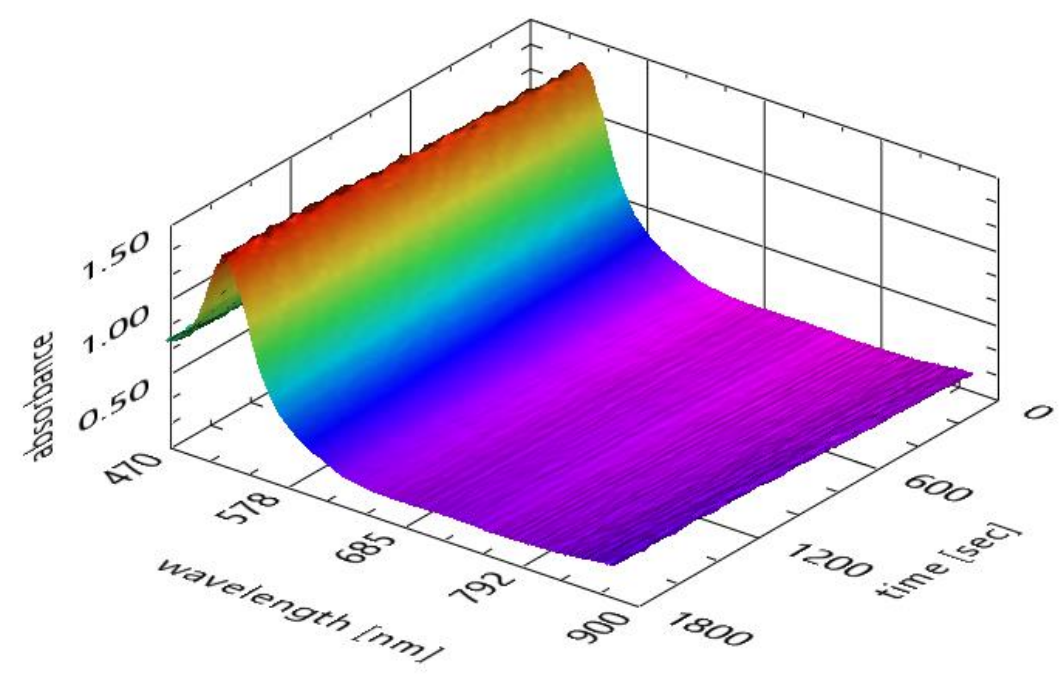

Figure S 11: UV-vis-NIR spectra measured simultaneously to the heterogeneous nucleation experiments with a PSS functionalized surface under the light microscope. The nanoparticle solution stays stable over the 40 minutes measurement time.

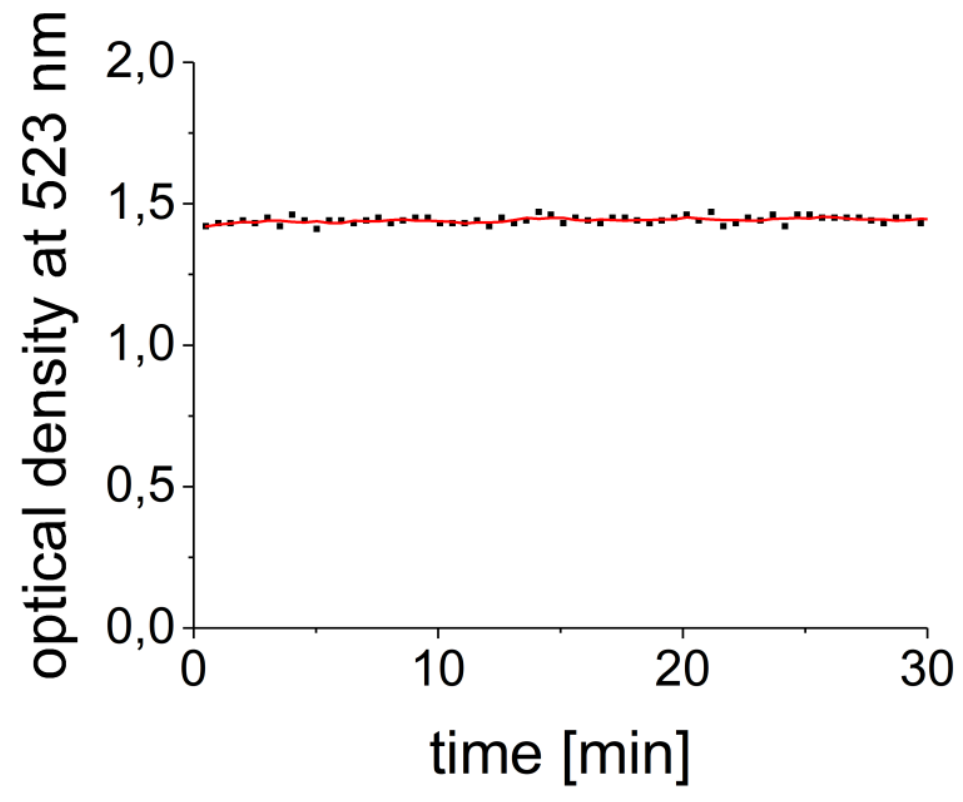

Figure S 12: Intensity at $523 \mathrm{~nm}$ of the UV-vis-NIR measurements performed simultaneously to the heterogeneous nucleation experiments with a PSS functionalized surface under the light microscope. The intensity is plotted against the experiment time and stays constant over the whole time period. 


\section{Materials}

Mica sheets were obtained from Micro to Nano in different shapes with a thickness of 0,15 to $0,21 \mathrm{~mm}$ and the highest grade V-1 quality. For ellipsometry measurements, double polished Si wafers (Orientation [100] of $5 \mathrm{~mm}$ length and $7 \mathrm{~mm}$ width) from Siegert Wafer were used.

All chemicals were purchased and used without further purification. Propan-2-ol, ethanol, acetone and hydrochloric acid (37\%) were purchased from VWR. Nitric acid was purchased from Carl Roth.

Hydrogen tetrachloroaurate trihydrate $\left(\mathrm{HAuCl}_{4} \cdot 3 \quad \mathrm{H}_{2} \mathrm{O}, \geq 99.9 \%\right)$, hexadecyltrimethylammonium bromide ( $C T A B \geq 99 \%$ ), cetyltrimethylammonium chloride solution (CTAC, 25 wt.- $\%$ in $\mathrm{H}_{2} \mathrm{O}$ ) and hexadecylpyridinium chloride monohydrate (CPC, 99.0 - 102.0\%) were purchased from Sigma-Aldrich. Sodium borohydride $\left(\mathrm{NaBH}_{4}, \geq 97 \%\right)$ and $\mathrm{L}(+)$-ascorbic acid $(\mathrm{AA}, \geq 99 \%$,) were purchased from Roth.

(3-Aminopropyl)triethoxysilane (APTES) for the amine functionalization was purchased from SigmaAldrich. (Heptadecafluoro-1,1,2,2-tetrahydrodecyl)triethoxysilane for the fluorine functionalization was purchased from abcr. N,N-Diisopropylethylamine (DIPEA) and Poly(sodium 4-styrenesulfonate) (PSS) with a $\mathrm{M}_{\mathrm{w}}$ of $70000 \mathrm{~g} / \mathrm{mol}$ were purchased from Sigma-Aldrich.

In all experiments Milli-Q water $(18,2 \mathrm{M} \Omega \mathrm{cm})$ was used.

\section{Instruments}

Transmission electron microscopy images were recorded on a Zeiss Libra 120 microscope at an accelerating voltage of $120 \mathrm{kV}$. The samples were applied on Quantifoil carbon-coated Cu 400 mesh grids.

AFM measurements were performed on a JPK NanoWizard instrument in the intermittent contact mode using a Bruker OTESPA-R3 silicon tip with a force constant of $26 \mathrm{Nm}^{-1}$ and a resonance frequency of about $300 \mathrm{kHz}$. Height, phase and amplitude images were recorded simultaneously.

For contact angles and free surface energies, the drop shape analyzer DSA25 and the software Advance from Krüss were used.

To determine the layer thickness the SENresearch 4.0 ellipsometer from SENTECH instruments GmbH was used.

Scanning electron microscopy images were recorded with a Gemini500 by Zeiss operating at $3 \mathrm{kV}$ equipped with an Inlens and a SE detector for secondary and backscattered electrons. Samples were sputter coated with an 2,5 nm platinum layer, mounted on aluminum stubs and attached by carbon conductive tabs.

UV-vis-NIR measurements were performed with a Varian Cary 50 spectrometer in quartz cuvettes. The UV-vis-NIR measurements carried out simultaneously with light microscopy measurements were performed with a modular USB2000+ spectrometer from Ocean Optics equipped with a USB-DT miniature deuterium tungsten halogen lamp.

Light microscopy images were recorded with an Axiolmager from Zeiss with an LD Epiplan 50x/0,50 HD DIC objective using transmitted light, bright field illumination, a condenser numerical aperture at 0,9 , a resolution of $0,3 \mu \mathrm{m}$, an Axiocam $506 \mathrm{bw}$ as an imaging device and an exposure time of $10 \mathrm{~ms}$. To enable a constant focus on the mica surface during the experiments, we mark the surface with a small scratch. 


\section{Literature}

1. Crampton, N.; Bonass, W. A.; Kirkham, J.; Thomson, N. H., Formation of aminosilanefunctionalized mica for atomic force microscopy imaging of DNA. Langmuir 2005, 21 (17), 78847891.

2. Deegan, R. D.; Bakajin, O.; Dupont, T. F.; Huber, G.; Nagel, S. R.; Witten, T. A., Capillary flow as the cause of ring stains from dried liquid drops. Nature 1997, 389 (6653), 827.

3. Denkov, N. D.; Velev, O.; Kralchevsky, P.; Ivanov, I.; Yoshimura, H.; Nagayama, K., Twodimensional crystallization. Nature 1993, 361 (6407), 26.

4. Hu, H.; Larson, R. G., Evaporation of a sessile droplet on a substrate. J. Phys. Chem. B 2002, 106 (6), 1334-1344.

5. Mirkin, C. A.; Letsinger, R. L.; Mucic, R. C.; Storhoff, J. J., A DNA-based method for rationally assembling nanoparticles into macroscopic materials. Nature 1996, 382 (6592), 607.

6. Sturm, E. V.; Cölfen, H., Mesocrystals: past, presence, future. Crystals 2017, 7 (7), 207. 